\title{
TURBULENCE MODELING IN STENOSED CAROTID ARTERIES USING CFD
}

\author{
Ibrahim Mustafa ${ }^{1}$, Shagufta Ishtiaque ${ }^{2}$ X.Y. Xu ${ }^{3}$ and N.B. Wood ${ }^{3}$ \\ ${ }^{1}$ Department of Chemical Engineering Technology, Yanbu Industrial College, Royal Commission for Jubail and Yanbu, P.O. Box \\ 30436, Yanbu Industrial City 21 477, Kingdom of Saudi Arabia. \\ ${ }^{2}$ Department of Chemical Engineering, University of Karachi, Karachi, Pakistan \\ ${ }^{3}$ Department of Chemical Engineering and Technology, Imperial College, London, UK
}

\begin{abstract}
Various numerical simulations of blood flow through stenosed (partially blocked) carotid arteries have been performed in the past but most of them have been limited to laminar flow. It has been reported in the literature that stenosis may promote disturbed flow causing transition from laminar to turbulence [1]. The studies on turbulence development in the post-stenotic regions are rare and have been performed by using only one or two turbulence models. In this work several turbulence models were used and validated against experimental data by using CFD (Computational Fluid Dynamics) package Fluent 6.1. A stenosed geometry of $75 \%$ area reduction was used in the simulation. It was found that SST (Shear Stress Transport) k-w model gave the best agreement with experimental data for Reynolds Number of 5000. We also analyzed the models at even lower Reynolds Number of 2000 and found that SST k-w model performed consistently better.
\end{abstract}

KEYWORDs: CFD, Modeling, Blood flow, Stehosed, Artries

\section{INTRODUCTION}

The build-up of arteriosclerotic plaque in carotid arteries may hamper blood flow to the brain due to reduced arterial area, and its rupture, with the release of plaque contents may cause thromboses to occur distally, leading to stroke - a major cause of death and disability in the modern world. To circumvent this situation a surgical procedure, endarterectomy is often recommended in which the plaque is removed. Carotid geometry varies widely between subjects, and the resulting variations in haemodynamics, which influence plaque formation, leads to varying degrees of individual risk.

The in-vivo diagnostic techniques such as MRI and Doppler Ultrasound are usually employed to help the surgeon in deciding the treatment plan. However, used in combination with medical images, CFD techniques may be employed to analyze blood flow patterns. The method of CFD greatly assists in identifying areas of flow separation and recirculation, causing reduced or fluctuating wall shear stresses, which are favorable for plaque development and may also have a role in its subsequent rupture.

For most haemodynamics problems, turbulence needs not to be considered. However, with upstream laminar flow entering a constriction, the Reynolds number is raised and the retarding velocity field in the region downstream of the stenosis can become transitional or even turbulent depending on the flow conditions and severity of the stenosis. For example, with an arterial cross-sectional 
area reduction of around $80 \%$, transition to turbulence generally occurs [1].

A range of CFD studies have been applied to turbulent flow in arteries, including stenoses [1-8]. Out of several turbulence models available in the literature, $k-\omega$ and $k-\varepsilon$ have been the most common.

The objective of this work is to test the performance of several turbulence models, including the SST $\mathrm{k}-\omega$ model developed by Menter [9] at low Reynolds numbers such as 5000 and 2000 by comparing them against experimental work of Deshpande and Giddens [10] for stenosed geometry of $75 \%$ area reduction. A commercial CFD software Fluent 6.1 is employed to solve the flow equations. It should be noted that these turbulence models have been validated only for $\operatorname{Re}>10,000$ [9] and hence their adequacy in capturing transition region is still in question. Although flows with Reynolds No. 5000 and 2000 are not realistic flows in blood arteries, but from the standpoint of selecting a suitable turbulence model that could work at low-Re, the present studies could provide a guideline about whether or not these models could be used for the transition region. Detailed 3D and pulsatile flow analysis will be addressed in future work.

\section{MODEL DEVELOPMENT}

For modeling isothermal fluid flow through constrained sections the conservation principles of mass and momentum in a specified domain must be satisfied. If turbulence is taken into account via an effective eddy viscosity, then time-averaged equations of continuity and motion for steady state flow take the following general form (Reynolds Equations):

$$
\begin{aligned}
& \nabla \bullet \bar{u}=0 \\
& \nabla \bullet \rho u u=-\nabla \bar{p}-\left[\nabla \bullet \bar{\tau}^{v}\right]-\left[\nabla \bullet \bar{\tau}^{R}\right]
\end{aligned}
$$

where $u$ is the velocity, $\mathrm{p}$ is the thermodynamic pressure, and $\tau$ is the stress tensor. The bar indicates that the quantity is time-averaged. The superscripts $v$ and $\mathrm{R}$ shows viscous and Reynolds stresses respectively. In case of a 2D axi-symmetric geometry the significant viscous stress would be $\tau_{\mathrm{rz}}$ given as follows:

$$
\bar{\tau}_{r z}{ }^{v}=-\mu \frac{d u_{z}}{d r}
$$

On the other hand, Reynolds stresses TR have to be estimated in order to close the system of equations. Several approaches have been presented in the past to model Reynolds stresses or turbulent momentum flux. Those include one-equation closure models such as Boussinesq's approximation, Prandtl mixing length, and Spalart Allamaras as well as twoequation closure models like $\mathrm{k}-\varepsilon, \mathrm{k}-\omega$, and SST models.

The selection of an appropriate turbulence model for a given application depends on several factors such as flow under considerations, $\mathrm{CPU}$ time, and degree of accuracy required. The commercial CFD software Fluent 6.1 has number of turbulence models available out of which we decided to check the performance of two-equation turbulence models such as realizable $\mathrm{k}-\varepsilon$ (RKE, Realizable K-E), $\mathrm{k}-\omega$, and SST-k- $\omega$ for simulation of flow through a stenosis. The latter two models are low-Re number models, whilst RKE is a modified version of standard $\mathrm{k}-\varepsilon$ to overcome latter's deficiency in separated flows. A brief review of selected models, without going into mathematical details is given next.

\subsection{K- $\varepsilon$ MODEL}

Different versions of $k-\varepsilon$ exist such as standard, low-Re, RNG(Renormalization Group) and Realizable. All these are semi-empirical models based on transport equations for turbulent kinetic energy $(\mathrm{k})$ and dissipation rate $(\varepsilon)$. 
The equation for $\mathrm{k}$ is derived mathematically where equation for $\varepsilon$ is generally based on physical reasoning. The major differences between these versions are the method of calculating turbulent viscosity, and generation and destruction terms in the equation for $\varepsilon$.

In deriving standard $\mathrm{k}-\varepsilon$ model a fully turbulent flow has been assumed therefore it is strictly valid for fully turbulent flows and therefore it is unsuitable for our purpose.

In RNG $\mathrm{k}-\varepsilon$ model the technique of Rnormalization Group is utilized which results in constants different from those in standard $\mathrm{k}-\varepsilon$ and additional terms and functions in the transport equations for $\mathrm{k}$ and $\varepsilon$. The RNG model is more responsive to the effects of rapid strain and streamline curvature than the standard $\mathrm{k}-\varepsilon$ model, which explains the superior performance of the RNG model for certain classes of flows [11].

The Realizable $\mathrm{k}-\varepsilon$ was developed to satisfy certain mathematical constraints to normal stresses consistent with the physics of turbulent flows. This model addresses the deficiencies of standard $\mathrm{k}-\varepsilon$ by adopting a new eddy-viscosity formula and a new model equation for dissipation rate. This model has been extensively validated for a wide range of flows including rotating homogeneous shear flows, free flows including jets and mixing layers, channel and boundary layer flows, and separated flows [12]. For all these cases, the performance of the model has been found to be substantially better than that of the standard k- $\varepsilon$ model.

In view of the above therefore, we decided to include the RKE version of $k-\varepsilon$ in our comparison.

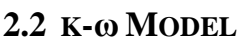

This is again an empirical model based on transport equations for $\mathrm{k}$ and $\omega$ where $\omega$ is the specific dissipation rate defined as the ratio of $\mathrm{k}$ and $\varepsilon$. This model is well known for low-
Re flows i.e., it calculates the flow down to the wall without the use of wall functions, and has been found very satisfactory in case of free shear flows and separated flows.

This revised, low-Re model was suggested by Wilcox in $1988[13,14]$ in which an equation related to the frequency $(\omega-\varepsilon / k)$ of eddy fluctuations was preferred over using an equation describing the turbulent dissipation rate $\varepsilon$. The new transport equation gives the advantage of accurately predicting the turbulent length scale in difficult-to-solve pressure gradient flows, leading to enhanced wall shear stress and heat transfer predictions. As with other low-Re treatments, the use of wall functions was discarded; additionally the wall damping function used in low-Re $\mathrm{k}-\varepsilon$ models was unnecessary.

The disadvantage of the model is that it is sensitive to the free stream values of $\omega$. A modification was suggested by Menter [9] in which $\mathrm{k}-\omega$ model was used in the inner boundary layer whereas $\mathrm{k}-\varepsilon$ model was used in the bulk flow. The modification resulted in another two-equation low-Re turbulence model known as SST $(k-\omega)$ which is discussed next.

\subsection{SST (K- $\omega)$ MODEL}

This model is considered as a variation of standard k- $\omega$ model. In this model, definition of the turbulent viscosity is modified to account for the transport of the principal turbulent shear stress. Other modifications include the addition of a cross-diffusion term in the $\omega$ equation and a blending function to ensure that the model equations behave appropriately in both the near-wall and farfield zones. These modifications make SST model to perform better than both the $k-\omega$ and the $k-\varepsilon$ models in a wide range of applications.

To the best of our knowledge, this model has not been validated in the range of low-Re 
turbulence to transition region with phenomena such as adverse pressure gradients and vortex shedding generally occurring in stenosed arteries.

The experimental data of Deshpande and Giddens [10], who conducted flow measurements for water flowing through a constricted 2 inch-dia tube with $75 \%$ area reduction, was used to validate the above models. The contour of constriction was chosen to be of cosine shape. In their studies, a Reynolds number range of 500 to 15000 was employed at the entrance of the tube. However, in this work we studied the model performance for Reynolds Number of 5000, 2000 and 1000 only. Studies at further low Reynolds Number will be discussed in the forthcoming paper.

\section{BOUNDARY CONDITIONS}

We have applied the no slip boundary condition at the wall, fully developed steady state velocity profile at the tube inlet, zero velocity gradient at the axis of symmetry and zero gauge pressure at the tube exit. A schematic of the stenosed-tube geometry considered is given in Fig. 1 along with all relevant dimensions.

The pre-stenotic length of $3 \mathrm{D}$, where $\mathrm{D}$ is the diameter of the tube, and post stenotic length of $16 \mathrm{D}$ were chosen. A short entrance length was considered suitable since all inlet boundary conditions used in this study are based on fully developed flow. An outlet length of $16 D$ was chosen to ensure that the outlet boundary conditions did not influence the flow in the vicinity of the stenosis.

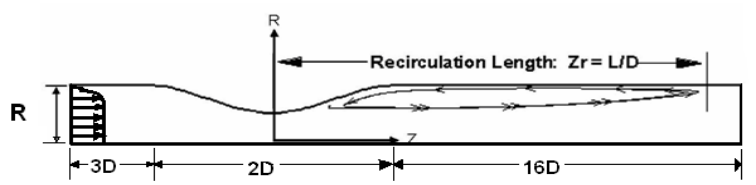

Fig. 1. Schematic of the Stenosed Geometry

\section{SOLUTION PROCEDURE}

The above set of governing equations and boundary conditions constitute a non-linear problem which can only be solved through numerical means. A well known CFD software Fluent 6.1 was, therefore, employed to carry out simulations.

\subsection{Grid Generation and Refinement}

The grid was built in Gambit 2.0., the preprocessor for Fluent, which discretized the domain into a number of quadrilateral elements. The discretization was maintained finer in the stenotic and post stenotic regions. To ensure grid independent results three different meshes with increasing number of nodes (coarse, medium and fine) were used. The grids output were compared with the experimental data of Despande and Giddens [10] at the center of stenosis as shown in Fig. 2.

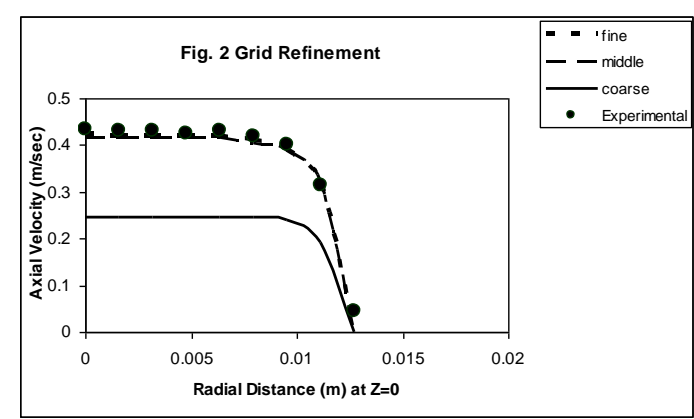

Fig. 2. Grid Sensitivity Analysis at the Center of Stenosis

Because the difference in output of the medium and fine meshes was within $1 \%$ we chose the middle mesh for our work. The mesh consisted of approximately 47,500 quadrilateral cells and 48501 nodes, shown in Fig.3, which was found to provide sufficient spatial resolution.

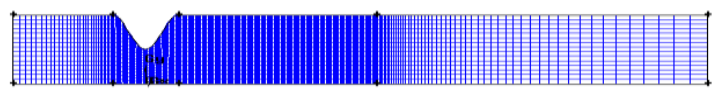

Fig.3. Medium Grid with $75 \%$ area reduction and 47500 quadrilateral cells. 


\subsection{NUMERICAL SCHEME}

A segregated, implicit scheme was used to solve the governing equations. Convective terms in the momentum and turbulence equations were discretized using a secondorder upwind scheme. The pressure term was discretized using a second-order scheme and the pressure and velocity terms were coupled using the SIMPLEC method. The residuals of each equation for all simulations were converged to $10^{-4}$. Since higher order discretization schemes were employed, reducing the residuals further was considered unnecessary.

\section{RESULTS \& DISCUSSIONS}

The objective of this work, as mentioned above, was to check the performance of some likely turbulence models in flow related to a stenosed artery, and to recommend a suitable model for further studies in the transition region. We, therefore, tested above mentioned models against selected experimental results of Deshpande and Giddens [10].

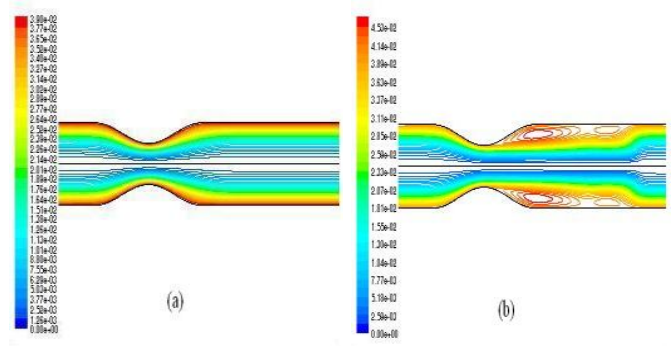

Fig. 4. Blood Profiles in in Stenosed Carotid Artery at two different Inlet Velocities.

In Figure 4, Fluent 6.1 output of blood profiles at (a) low velocity $(0.021 \mathrm{~cm} / \mathrm{sec})$ (b) high velocity $(0.025 \mathrm{~cm} / \mathrm{sec})$ are shown. As it can be seen, in case of a comparatively higher velocity, the formation of adversed pressure gradients and recirculations in the poststenotic region causing low shear stresses in the region and hence the probability of plaque formation. Further, in our first analyses we choose the center line axial velocity as a function of the axial distance as shown in Fig.5. It can be seen that the SST model gives better prediction than both $\mathrm{k}-\omega$ and $\mathrm{r}-\mathrm{k}-\varepsilon$ specially in the area of interest i.e., the post stenotic region.

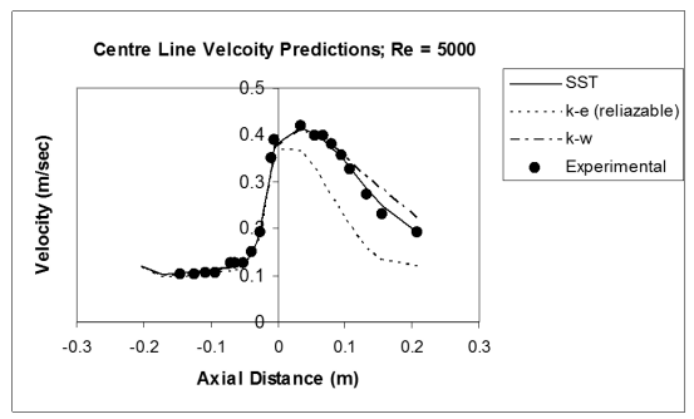

Fig.5. Center Line Axial Velocity as a Function of Axial Distance ( $\mathrm{z}=0$ is the center of Stenosis)

The $\mathrm{k}-\varepsilon$ model shows significant deviation from experimental data owing to its inability to capture low-Re physics near the wall in the viscous sub-layer. On the other hand, $\mathrm{k}-\omega$ model shows slight deviation in the poststenotic region probably due to sensitivity of the parameter $\omega$ under free stream conditions. In Fig. 6 velocity profile at the location of one tube diameter away from the center of stenosis is shown as a function of radial position. Both $\mathrm{k}-\omega$ and SST models predict well in the area which is also obvious from Fig. 5.

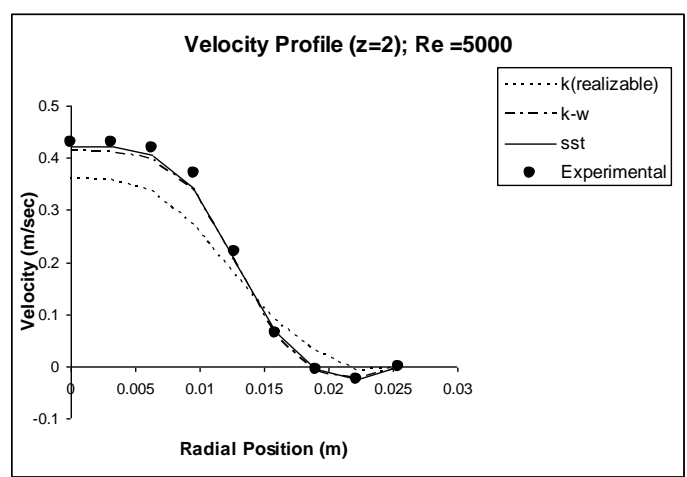

Fig. 6. Center Line Axial Velocity as a Function of radial distance at $\mathrm{z}=1 \mathrm{D}$ 
In Fig. 7 the prediction of reattachment length i.e., the extent of flow separation zone is plotted for all three models (arrow indicates experimentally determined reattachment length [10]). Here SST model again gives better result than the other two models.

We also conducted numerical studies for flows in the regime of $\mathrm{Re}=2000$ and compared our results with the experimental work of Deshande and Giddens [10]. This is shown in Fig. 8 below. As it can be seen. the shear stress is highest at the center of stenosis $(\mathrm{z} / \mathrm{D}=$ 0 ) and falls rapidly in near post-stenotic region. The maximum shear stress was estimated to be about $58 \mathrm{~N} / \mathrm{m}^{2}$ which was not recorded by the experimental work.

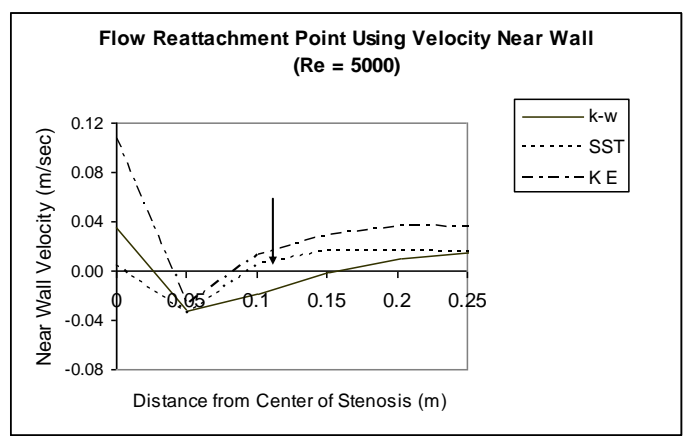

Fig.7. Estimation of Reattachment Length

However, again an excellent match with the experimental data was obtained.

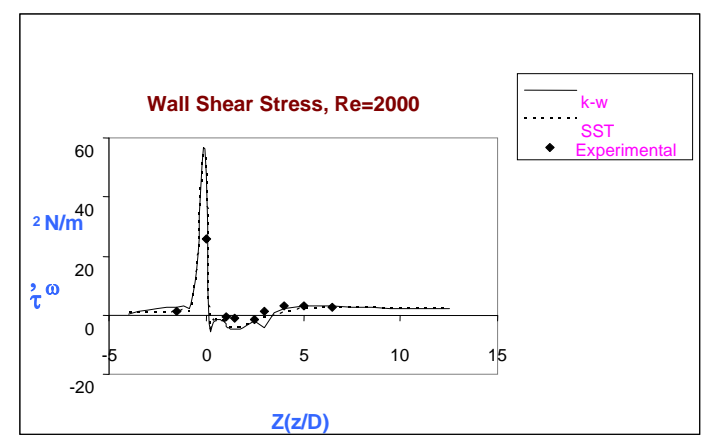

Fig.8. Models predictions at $\mathrm{Re}=2000$

\section{CONCLUSION}

From the results discussed above it is quite obvious that the SST k-w model has advantage over the standard $\mathrm{k}-\omega$ model. However, it seems necessary to further test these two models for a range of Reynolds numbers and under pulsatile flow conditions before picking up one for the simulation.

As we see, the computer software Fluent successfully predicted important parameters for the development of arteriosclerosis such as shear stress, recirculation, adverse pressure gradients and vortex formations. Areas of low shear stress and extent of recirculation which are prone to develop arteriosclerosis matched with high accuracy to those measured from experimental data. It should be noted that the prediction of velocities very near to the wall, where MRI etc. are plagued by noise, was well estimated by CFD. Although experiment data collected used water as a fluid but exactly the same approach can be adopted for blood flow through arteries. This can be done by taking in-vivo data from real patients and feed that to the same model and simply changing the fluid properties from water to blood.

In this preliminary work a very simplified case of $2 \mathrm{D}$ steady state model with smooth contours was considered. In reality however, the blood flow is $3 \mathrm{D}$ pulsatile and hence unsteady. Moreover, the rigid wall condition is not applicable in real cases since artery walls are flexible. The incorporation of these phenomena would yield better predictions as to the development of plaque and its rupture.

\section{REFERENCES}

[1] Wood N.B. and Xu X.Y. "Turbulence in Stenosis: Survey and Early Results", Proceedings of the Fourth International Conference on Fluid Mechanics, Dalian, China, July 28-31, 2001.

[2] Ku D N. "Blood Flow in Arteries", Annual Review Fluid Mech, Vol. 29, 1997, pp. 399-434. 
[3] Akhavan R, Kamm RD, Shapiro AH. "An Investigation of Transition to Turbulence in Bounded Oscillatory Stokes Flows", Part 2 Numerical Simulations, J. Fluid Mech, Vol.225, 1991, pp. 423-444.

[4] Ryval, J., Straatman, A.G. and Steinman, D.A., "Two-equation turbulence modeling of pulsatile flow in a stenosed tube", Journal of Biomechanical Engineering, Vol. 126 (5), 2004, pp. 625-635.

[5] Chein K-Y., "Predictions of Channel and Boundary-Layer Flows with a Low-ReynoldsNumber Turbulence Model”, AIAA Journal, Vol. 20, 1982, pp. 33-38.

[6] Durbin P.A., "Separated Flow Computations with the k- $\varepsilon$-y2Model”, AIAA Journal, Vol. 33(4), 1995, pp. 659-664.

[7] Ghalichi F., Deng X., De Champlain A., Douville Y., King M., Guidoin R., "Low Reynolds Number Turbulence Modeling of Blood Flow in Arterial Stenoses", Biorheology, Vol. 35, 1998, pp. 281-294.

[8] Goldberg, U.C., "Separated Flow Treatment with a New Turbulence Model”, AIAA Journal, Vol. 24, , 1986, pp. 899-908.

[9] F. R. Menter, "Two-Equation Eddy-Viscosity Models for Transonic Flows", AIAA Journal, Vol.32, 1994, pp. 1598-1605.

[10] Deshpande, M.D. and Giddens, D.P. "Turbulence Measurements in a Constricted Tube”, Journal of Fluid Mech. Vol. 97 (1), 1980, pp. 65-89.

[11] Yakhot V and Orszag SA., "Renormalization group theory of turbulence: I. Basic theory", Sci. Comput., Vol. 1, 1986, pp. 3-51.

[12] Fluent User's Guide.

[13] Shih T-H, Liou W. W., Shabbir, A., Yang, Z., Zhu, J. "A new k- $\varepsilon$ eddy viscosity model for high Reynolds number turbulent flows", Computers Fluids, Vol. 24, 1995, pp. 227-238.

[14] Wilcox D.C., "Simulation of Transition with a Two-Equation Turbulence Model”, AIAA Journal, Vol. 32(2), 1994, pp. 247-255. 


\title{
نمذجة سريان الام الإضظرابي في الثرايين المسدودة جزئياً بإستخدام ميكانيكا الموائع الحسابية
}

\author{
إبراهيم مصطفى1، شاقوفتا إشتياق2، س.ي.كتشو3، نايل وود33 \\ 1 قسم تقنية الهندسة الكيميائية، كلية ينبع الصناعية، المملكة العربية السعودية \\ 2 قسم الهندسة الكيميائية، جامعة كر اتثي، كر اتثي، الباكستان

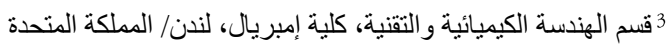

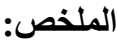

هناك دراسات عديدة في السابق لنمذجة سريان الدم في الثرايين المسدودة جزئياً ولكنها تتعلق فقط بالسريان الطبقي (على سرعات قليلة). ولقد أثبتت الدراسات السابقة بأنه في الثرايين المسدودة جزئياً يتم الإنتقال من السريان الطبقي إلى السريان الإضطر ابي، ولكن الدراسات المتعلقة بالسريان الإضطر ابي كانت محدودة فقط بنموذج واحد أو نموذجين ـ في هذه الدراسة تم إستخدام برنامج (Fluent 6.1) في ميكانيكا المو ائع الحسابية لنمذجة سريان الدم الإضطر ابي في الثرايين المسدودة جزئياً وقد نم إفتراض نسبة إنسداد قدرها 75\% في الحسابات وقد توصلنا إلى مطابقة نتائج الحسابات بإستخدام نموذج (SST K-W) مع النتائج المخبرية الحقيقية لرقم رينولا 5000 ـ كذلك تم تحليل النتائج على رقم رينولد 2000 ووجد أن مطابقة نتائج الحسابات مع التحاليل

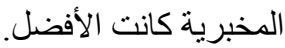

